Supporting Information

\title{
Single Molecular Reaction of Water on a ZnO Surface
}

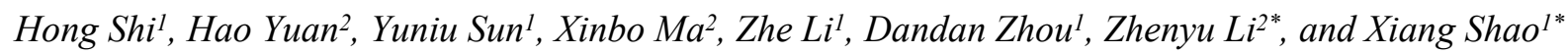

1. Department of Chemical Physics, CAS Key Laboratory of Urban Pollutant Conversion, Synergetic

Innovation Center of Quantum Information and Quantum Physics, University of Science and

Technology of China. 96 Jinzhai Road, Hefei, Anhui Province, 230026, China. E-mail:

shaox@ustc.edu.cn

2. Hefei National Laboratory for Physical Sciences at the Microscale, Synergetic Innovation Center of Quantum Information and Quantum Physics, University of Science and Technology of China. 96 Jinzhai Road, Hefei, Anhui Province, 230026, China. E-mail: zyli@ustc.edu.cn.
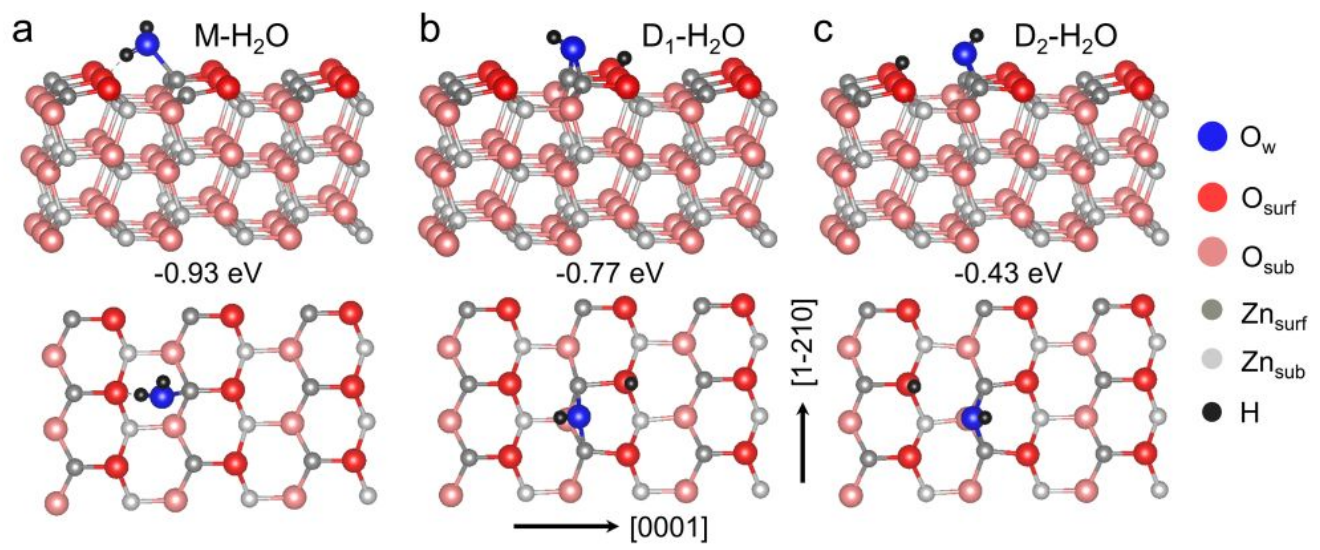

Figure S1. Adsorption models of single water on the $\mathrm{ZnO}(10-10)$ surface predicted by DFT calculations. (a) $\mathrm{M}-\mathrm{H}_{2} \mathrm{O}$, (b) D1- $\mathrm{H}_{2} \mathrm{O}$ and (c) $\mathrm{D} 2-\mathrm{H}_{2} \mathrm{O}$. 


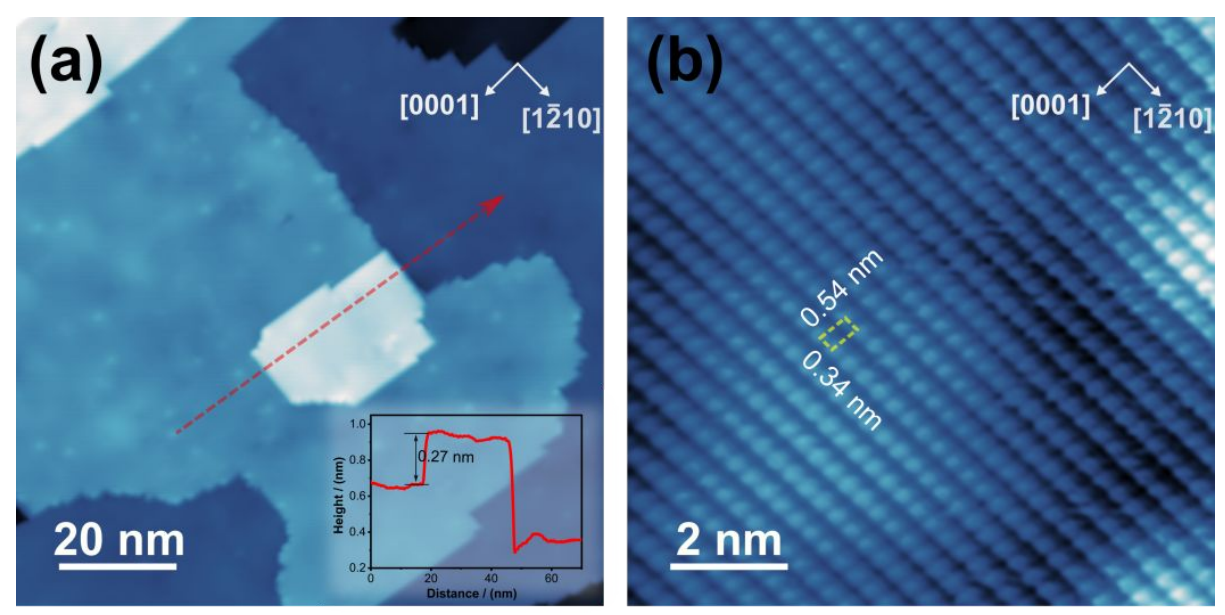

Figure S2. STM images of the as-prepared clean $\mathrm{ZnO}(10-10)$ surface. (a). STM image of the as-prepared clean $\mathrm{ZnO}(10-10)$ surface $(100 \mathrm{~nm} \times 100 \mathrm{~nm})$. Inset is the height profile along the red dashed line in (a). Image condition: $\mathrm{U}=3.2 \mathrm{~V}, \mathrm{I}=100 \mathrm{pA}$. (b). Atomically resolved STM image acquired with $\mathrm{U}=2.6 \mathrm{~V}, \mathrm{I}$ $=2.5 \mathrm{nA}(10 \mathrm{~nm} \times 10 \mathrm{~nm})$. It is generally accepted that under positive sample bias (empty state) the surface $\mathrm{Zn}$ lattice is imaged.

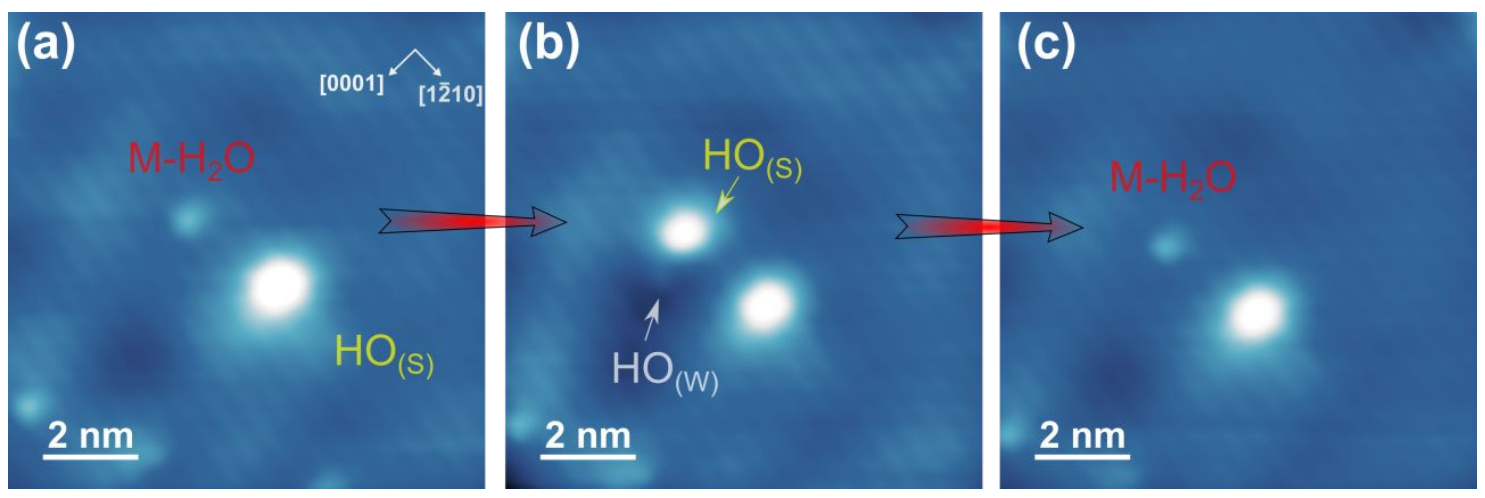

Figure S3. A STM sequence showing the structure evolution of a $\mathrm{M}-\mathrm{H}_{2} \mathrm{O}$ under STM tip-manipulation. (a) original state of $\mathrm{M}-\mathrm{H}_{2} \mathrm{O}$ and (b) after applying a negative pulse to form a $\mathrm{D} 2$ ' $-\mathrm{H}_{2} \mathrm{O}$. (c) Re-combination of $\mathrm{HO}_{(\mathrm{w})}$ and $\mathrm{HO}_{(\mathrm{S})}$ to form $\mathrm{M}-\mathrm{H}_{2} \mathrm{O}$ again after a push-manipulation with the STM tip. 


\section{- METHODS:}

\section{STM measurement:}

All the experiments were conducted on a commercial LT-STM (Createc Co.) housed in a UHV system equipped with a sample preparation chamber. The base pressure is better than $8.0 \times 10^{-11} \mathrm{mbar}$ for both STM and preparation chambers. The preparation of clean $\mathrm{ZnO}(10-10)$ surface and in-situ water exposing were carried out in preparation and STM chambers, respectively. All STM images were acquired in constant-current mode using an electrochemically etched gold tip at liquid nitrogen temperature. The $\mathrm{ZnO}(10-10)$ single crystals were supplied by Princeton Co. with a dimension of $10 \times 3 \times 0.5 \mathrm{~mm}^{3}$. The sample was cleaned through cycles of $2 \mathrm{keV} \mathrm{Ar}+$ sputtering and thermal annealing up to $1000 \mathrm{~K}$. To ensure the sample cleanness, usually the last annealing was finished in the STM chamber and then the sample was transferred into STM stage as fast as possible. Deionized water was purified by freeze-pump-thaw cycles before introducing into the UHV chamber via a high precision variable leak valve (VAT Co.). The water vapor was guided by a capillary that approaches close to a shutter-protected hole on the $\mathrm{LN}_{2}$-cooled STM shield. During exposing, the sample was kept in the STM stage and the tip was retracted for about $5 \mathrm{~mm}$ away for the sample surface.

The local barrier height (LBH) image was collected using the gap modulation method ${ }^{1,2}$. Tip-sample distance was modulated with a peak-to-peak amplitude of $0.05 \mathrm{~nm}$ and modulation frequency of $2 \mathrm{kHz}$. The modulated channel of the tunneling current is proportional to $\mathrm{dI} / \mathrm{dZ}$, where $\mathrm{I}$ and $\mathrm{Z}$ are the tunneling current and gap distance, respectively. Based on the formula ${ }^{3}: \Phi \approx 0.95(\mathrm{dI} / \mathrm{IdZ})^{\wedge} 2$. The $\mathrm{LBH}$ signal can be obtained from the output of the lock-in amplifier which collects the $\mathrm{dI} / \mathrm{dZ}$ signal. By recording $\mathrm{dI} / \mathrm{dZ}$ at each pixel, the LBH map can be collected simultaneously with the STM topography.

\section{Computational approach:}

DFT calculations were carried out by the projector-augmented wave (PAW) method ${ }^{4}$ using Vienna Ab initio Simulation Package $(\mathrm{VASP})^{5}$. The generalized gradient approximation (GGA) with Perdew-Burke-Ernzerhof (PBE) method ${ }^{6}$ was used to optimize the crystal structure of wurtzite ZnO bulk structure and the slabs. The plane-wave cutoff energy was set to $500 \mathrm{eV}$ and a $2 \times 2 \times 1 \Gamma$-centred k-point mesh was used for structural optimization (self-consistent). Surface slabs with a vacuum thickness of $15 \AA$ 
were modeled as the $\mathrm{ZnO}(10-10)$ surface. The three-layer of the $4 \times 3$ supercell was used as the terminal surface. All the structures were optimized until the force on each atom was smaller than $0.02 \mathrm{eV} \AA^{-1}$ and the convergence threshold for self-consistent iteration is $10^{-5} \mathrm{eV}$. The PDOS analysis was carried out to clarify the distributions of the molecular orbitals of water near the fermi level. The molecular orbitals were calculated using gaussian $16^{7}$ with M06-2X DFT methods ${ }^{8}$ and $6-311+\mathrm{G}(\mathrm{d}, \mathrm{p})$ basis set. The molecular graphics viewer VESTA was used to plot the crystal structures and charge densities. STM images were simulated based on a Tersoff-Hamann theory 9 .

\section{REFERENCES:}

(1) Ostermann, D.; Walther, G.; Schierbaum, K. D. Local-Barrier-Height Images of $\mathrm{TiO}_{2}(110)$ Surfaces. Phys. Rev. B - Condens. Matter Mater. Phys. 2005, 71, 1-10.

(2) Maeda, Y.; Okumura, M.; Tsubota, S.; Kohyama, M.; Haruta, M. Local Barrier Height of Au Nanoparticles on a $\mathrm{TiO}_{2}\left(\begin{array}{lll}1 & 1 & 0\end{array}\right)-\left(\begin{array}{l}1 \\ 1\end{array}\right.$ 2) Surface. Appl. Surf. Sci. 2004, 222, 409-414.

(3) Kobayashi, K.; Kurokawa, S.; Hasegawa, S.; Sakai, A. Local Tunneling Barrier Height at and around Subsurface Dopant Sites on P-GaAs(110). Jpn. J. Appl. Phys. 2010, 49, 1052011-1052016.

(4) Blöchl, P. E. Projector Augmented-Wave Method. Phys. Rev. B 1994, 50, 17953-17979.

(5) Kresse, G.; Hafner, J. Ab Initio Molecular Dynamics for Liquid Metals. Phys. Rev. B 1993, 47, 558-561.

(6) Perdew, J. P.; Burke, K.; Ernzerhof, M. Generalized Gradient Approximation Made Simple. Phys. Rev. Lett. 1996, 77, 3865-3868.

(7) Frisch, M.; Trucks, G.; Schlegel, H.; Scuseria, G.; Robb, M.; Cheeseman, J.; Scalmani, G.; Barone, V.; Petersson, G.; Nakatsuji, H. Gaussian 16. Gaussian, Inc. Wallingford, CT 2016.

(8) Zhao, Y.; Truhlar, D. G. The M06 Suite of Density Functionals for Main Group Thermochemistry, Thermochemical Kinetics, Noncovalent Interactions, Excited States, and Transition Elements: Two New Functionals and Systematic Testing of Four M06-Class Functionals and 12 Other Function. Theor. Chem. Acc. 2008, 120, 215-241.

(9) Tersoff, J.; Hamann, D. R. Theory of the Scanning Tunneling Microscope. Phys. Rev. B 1985, $31,805-813$. 